\title{
Homological Properties in Relation to Nakayama Algebras
}

\author{
Kwasi Baah Gyamfi, Abraham Aidoo, Dickson Y. B. Annor \\ Department of Mathematics, Kwame Nkrumah University of Science and Technology, Kumasi, Ghana \\ Email: kwasibaahgyamfi1@gmail.com, abramkhems09@gmail.com, dbannor1111@yahoo.com
}

How to cite this paper: Gyamfi, K.B., Aidoo, A. and Annor, D.Y.B. (2017) Homological Properties in Relation to $\mathrm{Na}$ kayama Algebras. Journal of Applied Mathematics and Physics, 5, 153-167. http://dx.doi.org/10.4236/jamp.2017.51016

Received: October 30, 2016

Accepted: January 22, 2017

Published: January 25, 2017

Copyright $\odot 2017$ by authors and Scientific Research Publishing Inc. This work is licensed under the Creative Commons Attribution International License (CC BY 4.0).

http://creativecommons.org/licenses/by/4.0/

\begin{abstract}
The goal of this paper is to investigate whether the Ext-groups of all pairs $(M, N)$ of modules over Nakayama algebras of type $(n, n, n)$ satisfy the condition $\operatorname{Ext}_{\Lambda}^{n}(M, N)=0$ for $n \gg 0 \Leftrightarrow \operatorname{Ext}_{\Lambda}^{n}(N, M)=0$ for $n \gg 0$. We achieve that by discussing the Ext-groups of Nakayama algebra with projectives of lengths $3 n+1$ and $3 n+2$ using combinations of modules of different lengths.
\end{abstract}

\section{Keywords}

Quivers, Path algebras, Ext-Groups, Projective Resolutions

\section{Introduction}

In this paper, we describe homological properties of Nakayama algebras. The algebra $\Lambda$ is a Nakayama algebra if every projective indecomposable and every injective indecomposable $\Lambda$-module is uniserial. In other words, these modules have a unique composition series, (see Schröer [1]). Nakayama algebras are finite dimensional and representation-finite algebras that have a nice representation theory in the sense that the finite-dimensional indecomposable modules are easy to describe.

The main contribution of this paper is to investigate whether the Ext-groups of all pairs $(M, N)$ of modules over Nakayama algebras of type $(n, n, n)$ satisfy the condition $\operatorname{Ext}_{\Lambda}^{n}(M, N)=0$ for $n \gg 0 \Leftrightarrow \operatorname{Ext}_{\Lambda}^{n}(N, M)=0$ for $n \gg 0$.

\section{Preliminary Notes}

This section will briefly discuss the properties of Nakayama algebra and some related propositions. We consider also proofs of some of the propositions. All the information presented here can be found in deeper details in the book from 
Rotman [2].

Definition: Let $\Lambda$ be an artin algebra. A $\Lambda$-module $A$ is called a uniserial module if the set of submodules is totally ordered by inclusion.

Proposition 1: The following are equivalent for $\Lambda$-module $A$

1. A is uniserial.

2. There is only one composition series for $A$.

3. The radical filtration of $A$ is a composition series for $A$.

4. The socle filtration of $A$ is a composition series for $A$.

5. $l(A)=r l(A)$, where $l(A)$ is the length of $A$ and $r l(A)$ is the radical length of $A$.

Proof: See Rotman [2] for the details.

Proposition 2: The following are equivalent for an artin algebra $\Lambda$.

1. $\Lambda$ is a sum of uniserial modules .

2. $\Lambda / a$ is a sum of uniserial modules for all ideals $a$ of $\Lambda$.

3. $\Lambda / r^{2}$ is a sum uniserial modules.

Proof: $1 \Rightarrow 2$ and $2 \Rightarrow 3$ are trivial. If $\Lambda$ is the sum of uniserial modules, then $\Lambda / a$ and $\Lambda / r^{2}$ which are factors of $\Lambda$ are also a sum of uniserial modules.

$$
3 \Rightarrow 1
$$

Let $P$ be an indecomposable projective $\Lambda$-module. We show that $P / r^{n} P$ is uniserial by induction on $n$ when $n \geq 2$. When $n=2$, there is nothing to prove. Suppose $n>2$. Let the radical filtration of $P$ be; $P \supset r P \supset r^{2} P \supset \cdots \cdots r^{n-1} P \supset r^{n} P=0$ such that $r^{i} P / r^{i+1} P$ is simple for $i=0,1, \cdots, n-1$.

When $n=3$, we have $r^{3} P \subset r^{2} P \subset r P \subset P$. Hence by induction hypothesis, $P / r^{n-1} P$ is uniserial. Considering the exact sequence $0 \rightarrow r P \rightarrow P \rightarrow P / r P \rightarrow 0$, which also implies that $P / r P$ is uniserial, hence $P / r^{n-1} P$ is also uniserial.

If $r^{n-1} P=0$, then $P / r^{n} P$ is clearly uniserial, so we have to assume that $r^{n-1} P \neq 0$. From proposition 1 , it follows that $r^{i} P / r^{i+1} P$ is simple for $i=0,1, \cdots, n-2$. To show that $P / r^{n} P$ is uniserial, then it is sufficient by proposition 1 to prove that $r^{n-1} \mathrm{P} / r^{n} \mathrm{P}$ is also simple.

Let $Q \rightarrow r^{n-2} P$ be a projective cover. Since $r^{n-2} P / r^{n-1 P}$ is simple, $Q$ must be indecomposable and so $Q / r^{2} Q$ is uniserial. But we have an epimorphism $r Q / r^{2} Q \rightarrow r^{n-1} P / r^{n} P$ which shows that $r^{n-1} P / r^{n} P$ is simple.

Proposition3: Proposition 2 (1).

Let $\varphi$ be a $D$ Tr-orbit of ind $\Lambda$. Suppose there is a projective module $P$ in $\varphi$. Then we have the following

1. $\varphi$ of non-zero objects in $\left\{P,\left(D T_{r}\right)^{-1} P, \cdots,\left(D T_{r}\right)^{-i} P, \cdots\right\}_{i \in N}$.

2. $\varphi$ is finite if and only if $\left(D T_{r}\right)^{-n} P=\left(T_{r} D\right)^{n}$ is injective for some $n$ in $N$. Moreover, if $\left(T_{r} D\right)^{n} P$ is injective, then $\varphi=\left\{P,\left(D T_{r}\right)^{-1} P, \cdots,\left(D T_{r}\right)^{-n} P\right\}$.

Proof: By proposition 1, $D \operatorname{Tr} P=0$ if and only if $P$ is projective. Since $P$ is projective module in $\varphi,(D T r)^{i} P=0$ for all $i>0$. Hence the claim in 2 (1).

We claim that if $(D T r)^{-i} P \simeq(T D r)^{-(i+j)} P \neq 0$ with $j>0$ we have $(D T r)^{i}(D T r)^{-(i+j)} P \simeq(D T r)(D T r)^{-i} P$ which implies that 
$P \simeq(D T r)^{j} P=(\operatorname{Tr} D)^{j} P$ which is not possible since $j>0 . \varphi$ can therefore be finite if $(D T r)^{-(n+1)} P=0$ for some $n \geq 0$. Since $(D T r)^{-n} P=(\operatorname{Tr} D)^{n} P$, then $P$ is injective in $\varphi$. We know therefore that if $(D T r)^{-n} P$ is injective, then $\varphi=\left\{P,(D T r)^{-1} P, \cdots,(D T r)^{-n} P\right\}$.

\section{Results}

The main goal of this work is to investigate whether the Ext-groups of all pairs $(\mathrm{M}, \mathrm{N})$ of modules over Nakayama algebras of type $(\mathrm{n}, \mathrm{n}, \mathrm{n})$ satisfy the condition: $\operatorname{Ext}_{\Lambda}^{n}(M, N)=0$ for $n \gg 0 \Leftrightarrow \operatorname{Ext}_{\Lambda}^{n}(N, M)=0$ for $n \gg 0$, where $n$ is a positive integer. We discuss the Ext-groups of Nakayama algebras with projectives of lengths $3 n+1$ and $3 n+2$ using combinations of modules of different lengths. Reader may refer to Auslander, $\mathrm{M}$ et al. [3] for ideas illustrated in this section.

We begin with the Ext-groups of Nakayama algebra with projectives of length $3 n+1$ using the combinations $(3 i, 3 j)$, and $(3 i, 3 j+2)$ where $3 i$ and $3 j$ are modules of lengths $3 i$ and $3 j$ respectively, $3 j+2$ is also module of length $3 j+2$.

Let $\Gamma$ be a path with the relations

$$
\alpha \gamma \beta \cdots \gamma \beta \alpha, \beta \alpha \gamma \cdots \alpha \gamma \beta \text { and } \gamma \beta \alpha \cdots \beta \alpha \gamma
$$

where the length of each relation is $3 n+1$. Let

$$
\Lambda=k \Gamma /\langle\alpha \gamma \beta \cdots \gamma \beta \alpha, \beta \alpha \gamma \cdots \alpha \gamma \beta, \gamma \beta \alpha \cdots \beta \alpha \gamma\rangle .
$$

The projectives of the above path algebra are as follows:

$$
P_{1}=\left(\begin{array}{c}
S_{1} \\
S_{2} \\
S_{3} \\
\vdots \\
S_{1}
\end{array}\right), \quad P_{2}=\left(\begin{array}{c}
S_{2} \\
S_{3} \\
S_{1} \\
\vdots \\
S_{2}
\end{array}\right), \quad P_{3}=\left(\begin{array}{c}
S_{3} \\
S_{1} \\
S_{2} \\
\vdots \\
S_{3}
\end{array}\right) .
$$

The above projectives $P_{1}, P_{2}$ and $P_{3}$ each has length $3 n+1$. The minimal projective resolution of the module $M=\left(S_{1}, S_{2}, \cdots, S_{3}\right)^{t}$ of length $3 i, i=1,2, \cdots, n$ is given as;

$$
\cdots Q_{4} \stackrel{d_{5}}{\longrightarrow} Q_{3} \stackrel{d_{4}}{\longrightarrow} Q_{3} \stackrel{d_{3}}{\longrightarrow} Q_{2} \stackrel{d_{2}}{\longrightarrow} Q_{1} \stackrel{d_{1}}{\longrightarrow} Q_{0} \stackrel{d_{0}}{\longrightarrow} M \rightarrow 0
$$

where $Q_{6 i}=Q_{6 i+1}=P_{1}, Q_{6 i+2}=Q_{6 i+3}=P_{2}$ and $Q_{6 i+4}=Q_{6 i+5}=P_{3}$ for $i \geq 0$.

The combinations have been reduced to four class because in the above minimal projective resolution of the module of length $3 i$, we see that it in the same group as the module of length $3 i+1$. The modules of lengths $3 i$ and $3 i+1$ therefore have the same properties with respect to the conditions; $\operatorname{Ext}_{\Lambda}^{n}(M, N)=0$ for $n \gg 0 \Leftrightarrow E x t_{\Lambda}^{n}=0$ for $n \gg 0$.

From the above resolution, we have;

$$
\begin{array}{r}
\cdots \Lambda e_{1} \stackrel{d_{6}}{\longrightarrow} \Lambda e_{3} \stackrel{d_{5}}{\longrightarrow} \Lambda e_{3} \stackrel{d_{4}}{\longrightarrow} \Lambda e_{2} \stackrel{d_{3}}{\longrightarrow} \lambda e_{2} \\
\stackrel{d_{2}}{\longrightarrow} \Lambda e_{1} \stackrel{d_{1}}{\longrightarrow} \Lambda e_{1} \stackrel{d_{0}}{\longrightarrow}\left(S_{1}, S_{2}, \cdots, S_{3}\right) \rightarrow 0 .
\end{array}
$$

Let $A=\left(S_{1}, S_{2}, \cdots, S_{3}\right)^{t}$, the pd $A=\infty$ since the resolution is periodic. The 
period is 6 . The truncation of the resolution is given as;

$P \cdots \Lambda e_{1} \stackrel{d_{6}}{\longrightarrow} \Lambda e_{3} \stackrel{d_{5}}{\longrightarrow} \Lambda e_{3} \stackrel{d_{4}}{\longrightarrow} \Lambda e_{2} \stackrel{d_{3}}{\longrightarrow} \lambda e_{2} \stackrel{d_{2}}{\longrightarrow} \Lambda e_{1} \stackrel{d_{1}}{\longrightarrow} \Lambda e_{1} \stackrel{d_{0}}{\longrightarrow} 0$.

The map $d_{6 i+1}$ is the multiplication by $(\gamma \beta \alpha)^{i}, d_{6 i+2}$ is the multiplication by $\alpha(\gamma \beta \alpha)^{n-i}, d_{6 i+3}$ is multiplication by $(\alpha \gamma \beta)^{i}, d_{6 i+4}$ is the multiplication by $\beta(\alpha \gamma \beta)^{n-i}, d_{6 i+5}$ is the multiplication by $(\beta \alpha \gamma)^{i}$ and $d_{6 i}$ is the multiplication by $\gamma(\beta \alpha \gamma)^{n-i}$.

Applying $\operatorname{Hom}_{\Lambda}(, M)$ where $M$ is the module

$$
\left(\begin{array}{c}
S_{1} \\
S_{2} \\
\vdots \\
S_{3}
\end{array}\right)
$$

of length $3 j$, we have;

$$
\begin{aligned}
& 0 \stackrel{d_{0}^{\times}}{\longrightarrow} \operatorname{Hom}_{\Lambda}\left(\Lambda e_{1}, M\right) \stackrel{d_{1}^{\times}}{\longrightarrow} \operatorname{Hom}_{\Lambda}\left(\Lambda e_{1}, M\right) \stackrel{d_{2}^{\times}}{\longrightarrow} \operatorname{Hom}_{\Lambda}\left(\Lambda e_{2}, M\right) \\
& \stackrel{d_{3}^{\times}}{\longrightarrow} \operatorname{Hom}_{\Lambda}\left(\Lambda e_{2}, M\right) \stackrel{d_{4}^{\times}}{\longrightarrow} \operatorname{Hom}_{\Lambda}\left(\Lambda e_{3}, M\right) \stackrel{d_{5}^{\times}}{\stackrel{H o m}{\Lambda}}\left(\Lambda e_{1}, M\right),
\end{aligned}
$$

where $\operatorname{Hom}_{\Lambda}\left(\Lambda e_{1}, M\right) \simeq e_{1} M, \operatorname{Hom}_{\Lambda}\left(\Lambda e_{2}, M\right) \simeq e_{2} M$ and $\operatorname{Hom}_{\Lambda}\left(\Lambda e_{3}, M\right) \simeq e_{3} M$.

We have the following Ext-groups;

$$
\operatorname{Ext}_{\Lambda}^{1}(N, M)=\operatorname{ker}\left(e_{1} M \stackrel{\alpha(\gamma \beta \alpha)^{n-i}}{\longrightarrow} e_{2} M\right) / \mathfrak{I}\left(e_{1} M \stackrel{(\gamma \beta \alpha)^{i}}{\longrightarrow} e_{1} M\right),
$$

where $N$ and $M$ are modules of length $3 i$ and $3 j$ respectively. We first compute the kernel for

$$
d_{2}^{\times}:\left(e_{1} M \stackrel{\alpha(\gamma \beta \alpha)^{n-i}}{\longrightarrow} e_{2} M\right)
$$

We have

$$
d_{2}^{\times}\left(\left(a_{1}, a_{2}, \cdots, a_{s}, a_{s+1}, \cdots\right)^{t}, 0,0\right)=\left(0,\left(0,0,0, \cdots, 0, a_{1}, a_{2}, \cdots, a_{s}\right)^{t}, 0\right)
$$

where $a_{1}$ is in the coordinate number $n-i+1$, therefore $a_{s}$ is in the coordinate number $n-i+s$. Hence $s=j+i-n$ and consequently $s+1=j+i+1-n$, $\left.\operatorname{ker}\left(e_{1} M \stackrel{\alpha(\gamma \beta \alpha)^{n-i}}{\longrightarrow} e_{2} M\right)=\left\{\left(\left(0,0, \cdots, 0, a_{s+1}, \cdots, a_{j}\right)\right)^{t}, 0,0\right) \mid a_{r} \in k, r=s+1, \cdots, j\right\}$.

Next we compute the image of

$$
d_{1}^{\times}:\left(e_{1} M \stackrel{(\gamma \beta \alpha)^{i}}{\longrightarrow} e_{1} M\right) .
$$

We have that

$$
d_{1}^{\times}\left(\left(b_{1}, b_{2}, \cdots, b_{j}\right)^{t}, 0,0\right)=\left(\left(0,0, \cdots, 0, b_{1}, \cdots, b_{j-1}\right)^{t}, 0,0\right)
$$

where $b_{1}$ is in the coordinate number $i+1$. This shows that

$$
\mathfrak{I}\left(e_{1} M \stackrel{(\gamma \beta \alpha)^{i}}{\longrightarrow} e_{1} M\right)=\left\{\left(\left(0,0, \cdots, 0, b_{1}, \cdots, b_{j-1}\right)^{t}, 0,0\right) \mid b_{t} \in k, t=1, \cdots, j-1\right\} .
$$

Hence $\operatorname{dim}_{k} \operatorname{Ext}_{\Lambda}^{1}\left(\left(S_{1}, S_{2}, \cdots, S_{3}\right)^{t}, M\right)=(i+1)-(j+i+1-n)=n-j$, for 
$j \leq n$. We compute

$\left.\operatorname{Ext}_{\Lambda}^{2}\left(\left(S_{1}, S_{2}, \cdots, S_{3}\right)^{t}, M\right)\right)=\operatorname{ker}\left(e_{2} M \stackrel{(\alpha \gamma \beta)^{i}}{\longrightarrow} e_{2} M\right) / \mathfrak{J}\left(e_{1} M \stackrel{\alpha(\gamma \beta \alpha)^{n-i}}{\longrightarrow} e_{2} M\right)$.

We compute the kernel for

$$
d_{3}^{\times}:\left(e_{2} M \stackrel{(\alpha \gamma \beta)^{i}}{\longrightarrow} e_{2} M\right)
$$

we have

$$
d_{3}^{\times}\left(0,\left(a_{1}, a_{2}, \cdots, a_{j}\right)^{t}, 0\right)=\left(0,\left(0,0, \cdots, 0, a_{1}, \cdots, a_{j-i}\right)^{t}, 0\right)
$$

where $a_{1}$ is the coordinate number $i+1$. This shows that

$$
\operatorname{ker}\left(e_{2} M \stackrel{(\alpha \gamma \beta)^{i}}{\longrightarrow} e_{2} M\right)=\left\{\left(0,\left(0,0, \cdots, 0, a_{1}, \cdots, a_{j}\right)^{t}, 0\right) \mid a_{r} \in k, r=1, \cdots, j\right\},
$$

where $a_{1}$ is in the coordinate number $j-i+1$. Next we compute the image of

$$
d_{2}^{\times}:\left(e_{1} M \stackrel{\alpha(\gamma \beta \alpha)^{n-i}}{\longrightarrow} e_{1} M\right) .
$$

We see that

$$
d_{2}^{\times}\left(\left(b_{1}, b_{2, \cdots, b_{s}, \cdots, b_{j}}\right)^{t}, 0\right)=\left(0,\left(0,0, \cdots, 0, b_{1}, b_{2}, \cdots, b_{s}\right)^{t}, 0\right)
$$

where $b_{1}$ is in the coordinate number $n-i+1$ and therefore $b_{s}$ is in the coordinate number $n-i+s$. This implies $n-i+s=j$ and hence $s=j+i-n$,

$$
\mathfrak{J}\left(e_{1} M \stackrel{\alpha(\gamma \beta \alpha)^{n-i}}{\longrightarrow} e_{2} M\right)=\left\{\left(0,\left(0,0, \cdots, 0, b_{1}, \cdots, b_{s}\right)^{t}, 0\right) \mid b_{t} \in k, t=1, \cdots, s\right\} .
$$

Hence $\operatorname{dim}_{k} \operatorname{Ext}_{\Lambda}^{2}\left(\left(S_{1}, S_{2}, \cdots, S_{3}\right)^{t}, M\right)=(n-i+1)-(j-i+1)=n-j$ for $j \leq n$.

$$
\operatorname{dim}_{k} \operatorname{Ext}_{\Lambda}^{2}\left(\left(S_{1}, \cdots, S_{3}\right)^{t}, M\right)=n-j
$$

We compute

$$
\operatorname{Ext}_{\Lambda}^{3}\left(\left(S_{1}, \cdots, S_{3}\right)^{t}, M\right)=\operatorname{ker}\left(e_{2} M \stackrel{\beta(\alpha \gamma \beta)^{n-i}}{\longrightarrow} e_{3} M\right) / \mathfrak{J}\left(e_{2} M \stackrel{(\alpha \gamma \beta)^{t}}{\longrightarrow} e_{2} M\right) \text {. }
$$

First we compute the kernel for

$$
d_{4}^{\times}:\left(e_{2} M \stackrel{\beta(\alpha \gamma \beta)^{n-i}}{\longrightarrow} e_{3} M\right) \text {. }
$$

We have

$$
d_{4}^{\times}\left(0,\left(a_{1}, a_{2}, \cdots, a_{s}, \cdots, a_{j}\right)^{t}, 0\right)=\left(0,0,\left(0,0, \cdots, 0, a_{1}, a_{2}, \cdots, a_{s}\right)^{t},\right),
$$

where $a_{1}$ is in the coordinate number $n-i+1$ and therefore $a_{s}$ is in the coordinate number $n-i+s$. Hence $s=j+i-n$ and consequently $s+1=j+i+1-n$. This shows that

$$
\begin{aligned}
& \operatorname{ker}\left(e_{2} M \stackrel{\beta(\alpha \gamma \beta)^{n-i}}{\longrightarrow} e_{3} M\right) \\
& =\left\{\left(0,\left(0,0, \cdots, 0, a_{s+1}, a_{s+2}, \cdots, a_{j}\right)^{t}, 0\right) \mid a_{r} \in k, r=s+1, \cdots, j\right\} .
\end{aligned}
$$

We compute the image of 


$$
d_{3}^{\times}:\left(e_{2} M \stackrel{(\alpha \gamma \beta)^{i}}{\longrightarrow} e_{2} M\right)
$$

to have

$$
d_{3}^{\times}\left(0,\left(b_{1}, b_{2}, \cdots, b_{j}\right)^{t}, 0\right)=\left(0,\left(0,0, \cdots, 0, b_{1}, b_{2}, \cdots, b_{j-i}\right)^{t}, 0\right)
$$

where $b_{1}$ is in the coordinate number $i+1$. This shows that

$$
\mathfrak{I}\left(e_{2} M \stackrel{(\alpha \gamma \beta)^{i}}{\longrightarrow} e_{2} M\right)=\left\{\left(0,\left(0,0, \cdots, b_{1}, \cdots, b_{j-i}\right)^{t}, 0\right) \mid b_{t} \in k, t=1, \cdots, j-i\right\} .
$$

Hence

$$
\operatorname{dim}_{k} \operatorname{Ext}_{\Lambda}^{3}\left(\left(S_{1}, \cdots, S_{3}\right)^{t}, M\right)=i+1-(j+i+1-n)=n-j
$$

for $j \leq n$.

Next we have

$$
\operatorname{Ext}_{\Lambda}^{4}\left(\left(S_{1}, \cdots, S_{3}\right)^{t}, M\right)=\operatorname{ker}\left(e_{3} M \stackrel{(\beta \alpha \gamma)^{i}}{\longrightarrow} e_{3} M\right) / \mathfrak{I}\left(e_{2} M \stackrel{\beta(\alpha \gamma \beta)^{n-i}}{\longrightarrow} e_{3} M\right)
$$

Similarly, we have

$$
\operatorname{ker}\left(e_{3} M \stackrel{(\beta \alpha \gamma)^{i}}{\longrightarrow} e_{3} M\right)=\left\{\left(0,0,\left(0,0, \cdots, 0, a_{1}, a_{2}, \cdots, a_{j}\right)^{t}, 0\right) \mid a_{r} \in k, r=1, \cdots, j\right\}
$$

where $a_{1}$ is in the coordinate number $j+1-i$, then

$$
\begin{gathered}
\mathfrak{I}\left(e_{2} M \underset{\beta(\alpha \gamma \beta)^{n-i}}{\longrightarrow} e_{3} M\right)=\left\{\left(0,0,\left(0,0, \cdots, 0, b_{1}, b_{2} \cdots, b_{s}\right)^{t}\right) \mid b_{t} \in k, t=1, \cdots, s\right\}, \\
s=j+i-n \text { where } b_{1} \text { is in the coordinate number } n-i+1 . \text { Hence } \\
\quad \operatorname{dim}_{k} \operatorname{Ext}_{\Lambda}^{4}\left(\left(S_{1}, \cdots, S_{3}\right)^{t}, M\right)=n-i+1-(j+1-i)=n-j, j \leq n .
\end{gathered}
$$

Again we have

$$
\operatorname{Ext}_{\Lambda}^{5}\left(\left(S_{1}, \cdots, S_{3}\right)^{t}, M\right)=\operatorname{ker}\left(e_{3} M \stackrel{\gamma(\beta \alpha \gamma)^{n-i}}{\longrightarrow} e_{1} M\right) / \mathfrak{I}\left(e_{3} M \stackrel{(\beta \alpha \gamma)^{i}}{\longrightarrow} e_{3} M\right) .
$$

We compute the kernel for

$$
d_{6}^{\times}:\left(e_{3} M \rightarrow e_{1} M\right)
$$

to have

$$
d_{6}^{\times}\left(0,0,\left(a_{1}, a_{2}, \cdots, a_{s}, \cdots, a_{j}\right)^{t}\right)=\left(\left(0,0, \cdots, 0, a_{1}, a_{2}, \cdots, a_{s}\right)^{t}, 0,0\right)
$$

where $a_{1}$ is in the coordinate number $n-i+2$ and therefore $a_{s}$ is in the coordinate number $n-i+1+s, n-i+s+1=j s=j+i-n-1$ and consequently $s+1=j+i-n$. This show that

$$
\operatorname{ker}\left(e_{3} M \rightarrow e_{1} M\right)=\left\{\left(0,0,\left(0,0, \cdots, a_{s+1}, \cdots, a_{j}\right)^{t}\right) \mid a_{r} \in k, r=s+1, \cdots, j\right\} .
$$

We compute the image for

$$
d_{5}^{\times}:\left(e_{3} M \stackrel{(\beta \alpha \gamma)^{i}}{\longrightarrow} e_{3} M\right)
$$

to get 


$$
d_{5}^{\times}\left(0,0,\left(b_{1}, b_{2}, \cdots, b_{j-i}\right)^{t}\right)=\left(0,0,\left(0,0, \cdots, 0, b_{1}, b_{2}, \cdots, b_{j-i}\right)^{t}\right)
$$

where $b_{1}$ is in the coordinate number $i+1$. This shows that

$$
\mathfrak{J}\left(e_{3} M \stackrel{(\beta \alpha \gamma)^{i}}{\longrightarrow} e_{3} M\right)=\left\{\left(0,0,\left(0,0, \cdots, b_{1}, \cdots, b_{j-i}\right)^{t}\right) \mid b_{t} \in k, t=1, \cdots, j-1\right\} .
$$

Hence $\operatorname{dim}_{k} \operatorname{Ext}_{\Lambda}^{5}\left(\left(S_{1}, \cdots, S_{3}\right)^{t}, M\right)=i+1-(j+i-n)=n+1-j$. This shows that $\operatorname{dim}_{k} \operatorname{Ext}_{\Lambda}^{t}(3 i, 3 j)=n-j$ for $t=1,2,3,4$ and $n-j+1$ for $t=5, n-j=0, n-j+1=0$ which implies $n=j$ and $n+1=j$.

It follows from the computation of the fifth Ext-group that the condition $\operatorname{Ext}_{\Lambda}^{n}(M, N)=0$ for $n \gg 0 \Leftrightarrow \operatorname{Ext}_{\Lambda}^{n}(N, M)=0$ for $n \gg 0$ holds.

We calculate the Ext-groups for the combination $(3 i, 3 j+2)$.

$$
\operatorname{Ext}_{\Lambda}^{1}\left(\left(S_{1}, \cdots, S_{3}\right)^{t}, M\right)
$$

where $M$ is the module of length $3 j+2$

$$
\operatorname{Ext}_{\Lambda}^{1}\left(\left(S_{1}, \cdots, S_{3}\right)^{t}, M\right)=\operatorname{ker}\left(e_{1} M \stackrel{\alpha(\gamma \beta \alpha)^{n-i}}{\longrightarrow} e_{2} M\right) / \Im\left(e_{1} M \stackrel{(\gamma \beta \alpha)^{i}}{\longrightarrow} e_{1} M\right) \text {. }
$$

We compute the kernel for

$$
d_{2}^{\times}:\left(e_{1} M \stackrel{\alpha(\gamma \beta \alpha)^{n-i}}{\longrightarrow} e_{2} M\right),
$$

we have

$$
d_{2}^{\times}\left(\left(a_{1}, a_{2}, \cdots, a_{s}, \cdots, a_{j+1}\right)^{t}, 0,0\right)=\left(0,\left(0,0, \cdots, 0, a_{1}, a_{2}, \cdots, a_{s}\right)^{t}, 0\right)
$$

where $a_{1}$ is in the coordinate number $n-i+1$ and therefore $a_{s}$ is in the coordinate number $n-i+s=j+1$. Hence $s=j+i-n$ and consequently $s+1=j+i+2-n$. This shows that

$$
\begin{aligned}
& \operatorname{ker}\left(e_{1} M \stackrel{\alpha(\gamma \beta \alpha)^{n-i}}{\longrightarrow} e_{2} M\right) \\
& =\left\{\left(\left(0,0, \cdots, 0, a_{s+1}, \cdots, a_{j+1}\right)^{t}, 0,0\right) \mid a_{r} \in k, r=s+1, \cdots, j+1\right\} .
\end{aligned}
$$

Computing the image for

$$
d_{1}^{\times}:\left(e_{1} M \stackrel{(\gamma \beta \alpha)^{i}}{\longrightarrow} e_{1} M\right)
$$

we have

$$
d_{1}^{\times}\left(\left(b_{1}, b_{2}, \cdots, b_{j+1}\right)^{t}, 0,0\right)=\left(\left(0,0, \cdots, 0, b_{1}, \cdots, b_{j+1-i}\right)^{t}, 0,0\right),
$$

where $b_{1}$ is in the coordinate number $i+1$. This shows that

$$
\mathfrak{J}\left(e_{1} M \stackrel{(\gamma \beta \alpha)^{i}}{\longrightarrow} e_{1} M\right)=\left\{\left(\left(0,0, \cdots, b_{1}, \cdots, b_{j+1-i}\right)^{t}, 0,0\right) \mid b_{t} \in k, t=1, \cdots, j+1-i\right\} .
$$

We therefore have

$$
\operatorname{dim}_{k} \operatorname{Ext}_{\Lambda}^{1}\left(\left(S_{1}, \cdots, S_{3}\right)^{t}, M\right)=i+1-(j+i+2-n)=n-(j+1)
$$

for $j+1 \leq n$. 
Next we have

$$
\operatorname{Ext}_{\Lambda}^{2}\left(\left(S_{1}, \cdots, S_{3}\right)^{t}, M\right)=\operatorname{ker}\left(e_{2} M \stackrel{(\alpha \gamma \beta)^{i}}{\longrightarrow} e_{2} M\right) / \mathfrak{I}\left(e_{1} M v e_{2} M\right) .
$$

We compute the kernel for

$$
d_{3}^{\times}:\left(e_{2} M \stackrel{(\alpha \gamma \beta)^{i}}{\longrightarrow} e_{2} M\right),
$$

we have

$$
d_{3}^{\times}\left(0,\left(a_{1}, a_{2}, \cdots, a_{j+1}\right)^{t}, 0\right)=\left(0,\left(0,0, \cdots, 0, a_{1}, \cdots, a_{j+1-i}\right)^{t}\right)
$$

where $a_{1}$ is in the coordinate number $i+1$. This shows that

$$
\operatorname{ker}\left(e_{2} M \stackrel{(\alpha \gamma \beta)^{i}}{\longrightarrow} e_{2} M\right)=\left\{\left(0,\left(0,0, \cdots, 0, a_{1}, \cdots, a_{j+1}\right)^{t}, 0\right) \mid a_{r} \in k, r=1, \cdots, j+1\right\} \text {, }
$$

where $a_{1}$ is in the coordinate number $j+2-i$. We compute the image for

$$
d_{2}^{\times}:\left(e_{1} M \stackrel{\alpha(\gamma \beta \alpha)^{n-i}}{\longrightarrow} e_{2} M\right) .
$$

We have

$$
d_{2}^{\times}\left(\left(b_{1}, b_{2}, \cdots, b_{s}, \cdots, b_{j+1}\right)^{t}, 0,0\right)=\left(0,\left(0,0, \cdots, b_{1}, b_{2}, \cdots, b_{s}\right)^{t}, 0\right)
$$

where $b_{1}$ is in the coordinate number $n-i+1$ and therefore $b_{s}$ is in the coordinate number $n-i+s=j+1$. Hence $s=j+1+i-n$. This shows that

$$
\mathfrak{J}\left(e_{1} M \stackrel{\alpha(\gamma \beta \alpha)^{n-i}}{\longrightarrow} e_{2} M\right)=\left\{\left(0,\left(0,0, \cdots, 0, b_{1}, \cdots, b_{j+1}\right)^{t}, 0\right) \mid b_{t} \in k, t=1, \cdots, j+1\right\} .
$$

We therefore have

$\operatorname{dim}_{k} \operatorname{Ext}_{\Lambda}^{2}\left(\left(S_{1}, \cdots, S_{3}\right)^{t}, M\right)=n-i+1-(j+2-i)=n-(j+1) \quad$ for $\quad j+1 \leq n$. Next we have

$$
\operatorname{Ext}_{\Lambda}^{3}\left(\left(S_{1}, \cdots, S_{3}\right)^{t}, M\right)=\operatorname{ker}\left(e_{2} M \stackrel{\beta(\alpha \gamma \beta)^{n-i}}{\longrightarrow} e_{3} M\right) / \mathfrak{I}\left(e_{2} M \stackrel{(\alpha \gamma \beta)^{i}}{\longrightarrow} e_{2} M\right) .
$$

Similarly, we have;

$$
\operatorname{ker}\left(e_{2} M \stackrel{\beta(\alpha \gamma \beta)^{n-i}}{\longrightarrow} e_{3} M\right)=\left\{\left(0,\left(0,0, \cdots, a_{1}, \cdots, a_{j+1}\right)^{t}, 0\right) \mid a_{r} \in k, r=1, \cdots, j+1\right\},
$$

where $a_{1}$ is in the coordinate number $j+i+2-n$. We also have

$$
\mathfrak{I}\left(e_{2} M \rightarrow e_{2} M\right)=\left\{\left(0,\left(0,0, \cdots, 0, b_{1}, \cdots, b_{j+1-i}\right)^{t}, 0\right) \mid b_{t} \in k, t=1, \cdots, j+1-i\right\},
$$

where $b_{1}$ is in the coordinate number $i+1$. We therefore have $\operatorname{dim}_{k} \operatorname{Ext}_{\Lambda}^{3}\left(\left(S_{1}, \cdots, S_{3}\right)^{t}, M\right)=i+1-(j+i+2-n)=n-(j+1)$ for $j+1 \leq n$. Next we have

$\operatorname{Ext}_{\Lambda}^{4}\left(\left(S_{1}, \cdots, S_{3}\right)^{t}, M\right)=\operatorname{ker}\left(e_{3} M \stackrel{(\beta \alpha \gamma)^{i}}{\longrightarrow} e_{3} M\right) / \mathfrak{I}\left(e_{2} M \stackrel{\beta(\alpha \gamma \beta)^{n-i}}{\longrightarrow} e_{3} M\right)$.

We compute the kernel for

$$
d_{5}^{\times}:\left(e_{3} M \stackrel{(\beta \alpha \gamma)^{i}}{\longrightarrow} e_{3} M\right) .
$$

We have 


$$
d_{5}^{\times}\left(0,0,\left(a_{1}, a_{2}, \cdots, a_{j}\right)^{t}\right)=\left(0,0,\left(0,0, \cdots, 0, a_{1}, \cdots, a_{j-i}\right)^{t}\right)
$$

where $a_{1}$ is in the coordinate number $i+1$. This shows that

$$
\operatorname{ker}\left(e_{3} M \stackrel{(\beta \alpha \gamma)^{i}}{\longrightarrow} e_{3} M\right)=\left\{\left(0,0,\left(0,0, \cdots, a_{1}, \cdots, a_{j}\right)^{t},\right) \mid a_{r} \in k, r=1, \cdots, j\right\},
$$

where $a_{1}$ is in the coordinate number $j+1-i$. We compute the image for

$$
d_{4}^{\times}:\left(e_{2} M \stackrel{\beta(\alpha \gamma \beta)^{n-i}}{\longrightarrow} e_{3} M\right) .
$$

We have

$$
d_{4}^{\times}\left(0,\left(b_{1}, b_{2}, \cdots, b_{s}, b_{j+1}\right)^{t}, 0,\right)\left(0,0\left(\left(0,0, \cdots, b_{1}, b_{2} \cdots, b_{s}\right)^{t}\right)\right.
$$

where $b_{1}$ is in the coordinate number $n-i+1$ and therefore $b_{s}$ is in the coordinate number $n-i+s=j+1$ and therefore $s=j+i-n$,

$$
\mathfrak{J}\left(e_{2} M \stackrel{\beta(\alpha \gamma \beta)^{i}}{\longrightarrow} e_{3} M\right)=\left\{\left(0,0,\left(0,0, \cdots, b_{1}, \cdots, b_{j-i}\right)^{t}\right) \mid b_{t} \in k, t=1, \cdots, j\right\} .
$$

We therefore have $\operatorname{dim}_{k} \operatorname{Ext}_{\Lambda}^{4}\left(\left(S_{1}, \cdots, S_{3}\right)^{t}, M\right)=n-i+1-(j+1-i) n-j$ for $j \leq n$. Finally, we have

$$
\operatorname{Ext}_{\Lambda}^{5}\left(\left(S_{1}, \cdots, S_{3}\right)^{t}, M\right)=\operatorname{ker}\left(e_{3} M \stackrel{\gamma(\beta \alpha \gamma)^{n-i}}{\longrightarrow} e_{1} M\right) / \Im\left(e_{3} M \stackrel{(\beta \alpha \gamma)^{i}}{\longrightarrow} e_{3} M\right) \text {. }
$$

We compute the kernel for

$$
d_{6}^{\times}:\left(e_{3} M \stackrel{\gamma(\beta \alpha \gamma)^{n-i}}{\longrightarrow} e_{1} M\right),
$$

we have

$$
d_{6}^{\times}\left(0,0,\left(a_{1}, a_{2}, \cdots, a_{s}, \cdots a_{j}\right)^{t}\right)=\left(\left(0,0, \cdots, 0, a_{1}, \cdots, a_{s}\right)^{t}, 0,0\right)
$$

where $a_{1}$ is in the coordinate number $n-i+2$ and therefore $a_{s}$ is in the coordinate number $n-i+1+s=j, s=j+i-n-1$ and consequently $s+1=j+i-n$. This shows that

$$
\operatorname{ker}\left(e_{3} M \stackrel{\gamma(\beta \alpha \gamma)^{n-i}}{\longrightarrow} e_{1} M\right)=\left\{\left(0,0,\left(0,0, \cdots, a_{1}, \cdots, a_{j}\right)^{t}\right) \mid a_{r} \in k, r=1, \cdots, j\right\} .
$$

We compute the image for

$$
d_{5}^{\times}:\left(e_{3} M \stackrel{(\beta \alpha \gamma)^{i}}{\longrightarrow} e_{3} M\right),
$$

we have

$$
d_{5}^{\times}\left(0,0,\left(b_{1}, b_{2}, \cdots, b_{j}\right)^{t}\right)=\left(0,0,\left(0,0, \cdots, 0, b_{1}, b_{2}, \cdots, b_{j-i}\right)^{t}\right)
$$

where $b_{1}$ is the ith coordinate number $i+1$ and hence

$$
\mathfrak{J}\left(e_{3} M \stackrel{(\beta \alpha \gamma)^{i}}{\longrightarrow} e_{3} M\right)=\left\{\left(0,0,\left(b_{1}, \cdots, b_{j-i}\right)^{t}\right) \mid b_{t} \in k, t=1, \cdots, j-i\right\} .
$$

We therefore have

$$
\operatorname{dim}_{k} \operatorname{Ext}_{\Lambda}\left(\left(S_{1}, \cdots, S_{3}\right)^{t}, M\right)=i+1-(j+i-n)=n+1-j \text { for } j \leq n+1
$$


$\operatorname{dim}_{k} \operatorname{Ext}_{\Lambda}^{t}(3 i, 3 j+2)=n-(j+1)$ for $t=1,2,3, n-j$ for $t=4$ and $n+1-j$ for $t=5$. We therefore have $n=j+1, n=j$ and $n=j-1$.

It follows from the computations of the forth and fifth Ext-groups that the condition $\operatorname{Ext}_{\Lambda}^{n}(M, N)=0$ for $n \gg 0 \Leftrightarrow \operatorname{Ext}_{\Lambda}^{n}(N, M)=0$ for $n \gg 0$ holds.

We now discuss the Ext-groups of the Nakayama algebras with the projectives of length $3 n+2$ using the combination $(3 i+1,3 j+1)$, where $3 i+1$ and $3 j+1$ are also modules of length $3 i+1$ and $3 j+1$ respectively.

Let $\Gamma$ be a path with the relations $\beta \alpha \gamma \cdots \gamma \beta \alpha, \gamma \beta \alpha \cdots \alpha \gamma \beta$ and $\alpha \gamma \beta \cdots \beta \alpha \gamma$ where the length of each relation is $3 n+2, n$ is a positive integer. Let

$$
\Lambda=k \Gamma /\langle\beta \alpha \gamma \cdots \gamma \beta \alpha, \gamma \beta \alpha \cdots \alpha \gamma \beta, \alpha \gamma \beta \cdots \beta \alpha \gamma\rangle \text {. }
$$

The projectives of the above path algebra are as follows:

$$
P_{1}=\left(S_{1}, S_{2}, \cdots, S_{1}, S_{2}\right)^{t}, P_{2}=\left(S_{2}, S_{3}, \cdots, S_{2}, S_{3}\right)^{t}, P_{3}=\left(S_{3}, S_{1}, \cdots, S_{3}, S_{1}\right)^{t} .
$$

The above projectives $P_{i}, i=1,2,3$ each has length $n+2$. The minimal projective resolution of module $\left(S_{1}, \cdots, S_{3}\right)^{i}$ of length $3 i$ is given as;

$$
\begin{aligned}
& \cdots Q_{6} \stackrel{d_{6}}{\longrightarrow} Q_{5} \stackrel{d_{5}}{\longrightarrow} Q_{4} \stackrel{d_{4}}{\longrightarrow} Q_{3} \stackrel{d_{3}}{\longrightarrow} Q_{2} \\
& \stackrel{d_{2}}{\longrightarrow} Q_{1} \stackrel{d_{1}}{\longrightarrow} Q_{0} \stackrel{d_{0}}{\longrightarrow} N \rightarrow 0 .
\end{aligned}
$$

where $N$ is the module of length $3 i, Q_{6 i}=Q_{6 i+1}=P_{1}, Q_{6 i+2}=Q_{6 i+3}=P_{3}$ and $Q_{6 i+4}=Q_{6 i+5}=P_{2}$ for $i \geq 0$ and $d_{6 i+1}$ is a multiplication by $(\gamma \beta \alpha)^{i}, d_{6 i+2}$ is a multiplication by $\beta \alpha(\gamma \beta \alpha)^{n-i}, d_{6 i+3}$ is a multiplication by $(\beta \alpha \gamma)^{i}, d_{6 i+4}$ is a multiplication by $\alpha \gamma(\beta \alpha \gamma)^{n-i}, d_{6 i+5}$ is a multiplication by $(\alpha \gamma \beta)^{i}$ and $d_{6 i}$ is multiplication by $\gamma \beta(\alpha \gamma \beta)^{n-i}$.

The combinations have been reduced to four class because in the above resolution, we see that the module of length $3 i$ is in the same group with the module of length $3 i+2$. The two modules therefore have the same properties with respect to the condition $\operatorname{Ext}_{\Lambda}^{n}(M, N)=0$ for $n \gg 0 \Leftrightarrow \operatorname{Ext}_{\Lambda}^{n}(N, M)=0$ for $n \gg 0$.

From the above resolution we have,

$$
\begin{gathered}
\cdots \Lambda e_{1} \stackrel{d_{7}}{\longrightarrow} \Lambda e_{2} \stackrel{d_{6}}{\longrightarrow} \Lambda e_{2} \stackrel{d_{5}}{\longrightarrow} \Lambda e_{3} \stackrel{d_{4}}{\longrightarrow} \Lambda e_{3} \\
\stackrel{d_{3}}{\longrightarrow} \Lambda e_{1} \stackrel{d_{2}}{\longrightarrow} \Lambda e_{1} \stackrel{d_{1}}{\longrightarrow} N \stackrel{d_{0}}{\longrightarrow} 0 .
\end{gathered}
$$

The $p d N=\infty$ since the resolution is periodic. The period is 6 . The truncation of the above resolution is given as:

$$
\begin{aligned}
& P \cdots \Lambda e_{1} \stackrel{d_{6}}{\longrightarrow} \Lambda e_{2} \stackrel{d_{5}}{\longrightarrow} \Lambda e_{2} \stackrel{d_{4}}{\longrightarrow} \Lambda e_{3} \\
& \quad \stackrel{d_{3}}{\longrightarrow} \Lambda e_{3} \stackrel{d_{2}}{\longrightarrow} \Lambda e_{1} \stackrel{d_{1}}{\longrightarrow} \Lambda e_{1} \stackrel{d_{0}}{\longrightarrow} 0 .
\end{aligned}
$$

Applying $\operatorname{Hom}(, M)$ where $M$ is the module of length $3 j$, we have

$$
\begin{aligned}
& 0 \stackrel{d_{0}^{\times}}{\longrightarrow} \operatorname{Hom}_{\Lambda}\left(\Lambda e_{1}, M\right) \stackrel{d_{1}^{\times}}{\longrightarrow} \operatorname{Hom}_{\Lambda}\left(\Lambda e_{1}, M\right) \stackrel{d_{2}^{\times}}{\longrightarrow} \operatorname{Hom}_{\Lambda}\left(\Lambda e_{3}, M\right) \\
& \stackrel{d_{3}^{\times}}{\longrightarrow} \operatorname{Hom}_{\Lambda}\left(\Lambda e_{3}, M\right) \stackrel{d_{4}^{\times}}{\longrightarrow} \operatorname{Hom}_{\Lambda}\left(\Lambda e_{2}, M\right) \stackrel{d_{5}^{\times}}{\longrightarrow} \operatorname{Hom}_{\Lambda}\left(\lambda e_{2}, M\right) \\
& \stackrel{d_{6}^{\times}}{\longrightarrow} \operatorname{Hom}_{\Lambda}\left(\Lambda e_{1}, M\right),
\end{aligned}
$$

where $\operatorname{Hom}_{\Lambda}\left(\Lambda e_{1}, M\right) \simeq e_{1} M, \operatorname{Hom}_{\Lambda}\left(\Lambda e_{2}, M\right) \simeq e_{2} M$ and 
$\operatorname{Hom}_{\Lambda}\left(\lambda e_{3}, M\right) \simeq e_{3} M$.

We discuss the Ext-groups of the Nakayama algebras with projectives of length $3 n+2$ by considering the case: $(3 i+1,3 j+1) .3 i+1$ is the module $\left(S_{1}, \cdots, S_{1}\right)^{i+1}$ of length $3 i+1$ and $3 j+1$ is the module $\left(S_{1}, \cdots, S_{1}\right)^{i+1}$ of length $3 j+1$.

The minimal projective resolution of the module $\left(S_{1}, \cdots, S_{1}\right)^{i+1}$ of length $3 i+1$ is given as;

$$
\begin{aligned}
\quad \cdots & \rightarrow Q_{6} \stackrel{d_{6}}{\longrightarrow} Q_{5} \stackrel{d_{5}}{\longrightarrow} Q_{4} \stackrel{d_{4}}{\longrightarrow} Q_{3} \stackrel{d_{3}}{\longrightarrow} Q_{2} \\
& \stackrel{d_{2}}{\longrightarrow} Q_{1} \stackrel{d_{1}}{\longrightarrow} Q_{0} \stackrel{d_{0}}{\longrightarrow} \rightarrow A \rightarrow 0
\end{aligned}
$$

where $Q_{6 i}=Q_{6 i+3}=P_{1}, Q_{6 i+1}=Q_{6 i+4}=P_{2}$ and $Q_{6 i+2}=Q_{6 i+5}=P_{3}$ for $i \geq 0, d_{6 i+1}$ is a multiplication by $\alpha(\gamma \beta \alpha)^{i+1}, d_{6 i+2}$ is a multiplication by $\beta(\alpha \gamma \beta)^{n-i}, d_{6 i+3}$ is a multiplication by $\gamma(\beta \alpha \gamma)^{i+1}, d_{6 i+4}$ is a multiplication by $\alpha(\gamma \beta \alpha)^{n-i}, d_{6 i+5}$ is a multiplication by $\beta(\alpha \gamma \beta)^{i+1}$ and $d_{6 i+6}$ is a multiplication by $\gamma(\beta \alpha \gamma)^{n-i}$ and $A$ is the module of length $3 i+1$.

Considering the above resolution, we have

$$
\begin{aligned}
& \cdots \rightarrow \Lambda e_{1} \stackrel{d_{6}}{\longrightarrow} \Lambda e_{3} \stackrel{d_{5}}{\longrightarrow} \Lambda e_{2} \stackrel{d_{4}}{\longrightarrow} \Lambda e_{1} \stackrel{d_{3}}{\longrightarrow} \Lambda e_{3} \\
& \stackrel{d_{2}}{\longrightarrow} \Lambda e_{2} \stackrel{d_{1}}{\longrightarrow} \Lambda e_{1} \stackrel{d_{0}}{\longrightarrow} A \rightarrow 0 \text {. }
\end{aligned}
$$

The $p \mathrm{~d} A=\infty$ since the resolution is periodic. The period ic 6 . The truncation of the above resolution is given as;

$$
\begin{aligned}
& P: \cdots \Lambda e_{1} \stackrel{d_{6}}{\longrightarrow} \Lambda e_{3} \stackrel{d_{5}}{\longrightarrow} \Lambda e_{2} \stackrel{d_{4}}{\longrightarrow} \Lambda e_{1} \\
& \stackrel{d_{3}}{\longrightarrow} \Lambda e_{3} \stackrel{d_{2}}{\longrightarrow} \Lambda e_{2} \stackrel{d_{1}}{\longrightarrow} \Lambda e_{1} \stackrel{d_{0}}{\longrightarrow} 0 .
\end{aligned}
$$

Applying $\operatorname{Hom}_{\Lambda}(, M)$, where $M$ is in the module $\left(S_{1}, \cdots, S_{j}\right)^{j}$ of length $3 j$, we have

$$
\begin{aligned}
& 0 \stackrel{d_{0}^{\times}}{\longrightarrow} \operatorname{Hom}_{\Lambda}\left(\Lambda e_{1}, M\right) \stackrel{d_{1}^{\times}}{\longrightarrow} \operatorname{Hom}_{\Lambda}\left(\Lambda e_{2}, M\right) \\
& \stackrel{d_{2}^{\times}}{\longrightarrow} \operatorname{Hom}_{\Lambda}\left(\Lambda e_{3}, M\right) \stackrel{d_{3}^{\times}}{\longrightarrow} \operatorname{Hom}_{\Lambda}\left(\Lambda e_{1}, M\right) \stackrel{d_{4}^{\times}}{\longrightarrow} \operatorname{Hom}_{\Lambda}\left(\Lambda e_{2}, M\right) \\
& \stackrel{d_{5}^{\times}}{\longrightarrow} \operatorname{Hom}_{\Lambda}\left(\lambda e_{3}, M\right) \stackrel{d_{6}^{\times}}{\longrightarrow} \operatorname{Hom}_{\Lambda}\left(\Lambda e_{1}, M\right),
\end{aligned}
$$

where $\operatorname{Hom}_{\Lambda}\left(\Lambda e_{1}, M\right) \simeq e_{1} M, \operatorname{Hom}_{\Lambda}\left(\Lambda e_{2}, M\right) \simeq e_{2} M$ and

$\operatorname{Hom}_{\Lambda}\left(\lambda e_{3}, M\right) \simeq e_{3} M$.

We compute the Ext-groups for $(3 i+1,3 j+1)$. The first Ext-group is;

$$
\operatorname{Ext}_{\Lambda}^{1}\left(\left(S_{1}, \cdots, S_{1}\right)^{i+1}, M\right)=\operatorname{ker}\left(e_{2} M \stackrel{\beta(\alpha \gamma \beta)^{n-i}}{\longrightarrow} e_{3} M\right) / \Im\left(e_{1} M \stackrel{\alpha(\gamma \beta \alpha)^{i+1}}{\longrightarrow} e_{2} M\right) \text {, }
$$

where $M$ is the module of length $3 j+1$. We compute the kernel for

$$
d_{2}^{\times}:\left(e_{2} M \stackrel{\beta(\alpha \gamma \beta)^{n-i}}{\longrightarrow} e_{3} M\right) .
$$

We have

$$
d_{2}^{\times}\left(0,\left(a_{1}, a_{2}, \cdots, a_{s}, \cdots, a_{j}\right)^{t}, 0\right)=\left(0,0,\left(0,0, \cdots, 0, a_{1}, \cdots, a_{s}\right)^{t}\right),
$$

where $a_{1}$ is in the coordinate number $n-i+1$ and therefore $a_{s}$ is in the coordinate number $n-i+s=j, s=j+i-n$ and consequently, $s+1=j+i+1-n$. 
This shows that

$$
\operatorname{ker}\left(e_{2} M \stackrel{\beta(\alpha \gamma \beta)^{n-i}}{\longrightarrow} e_{3} M\right)=\left\{\left(0,\left(0,0, \cdots, 0, a_{s+1}, \cdots, a_{j}\right)^{t}, 0\right) \mid a_{r} \in k, r=s+1, \cdots, j\right\} \text {. }
$$

We compute the image

$$
d_{1}^{\times}:\left(e_{1} M \stackrel{\alpha(\gamma \beta \alpha)^{i+1}}{\longrightarrow} e_{2} M\right) .
$$

We have

$$
d_{1}^{\times}\left(\left(b_{1}, b_{2}, \cdots, b_{j+1}\right)^{t}, 0,0\right)=\left(0,\left(0,0, \cdots, 0, b_{1}, \cdots, b_{j-(i+1)}\right)^{t}, 0\right),
$$

where $b_{1}$ is in the coordinate number $i+2$. Hence

$$
\mathfrak{I}\left(e_{1} M \stackrel{\alpha(\gamma \beta \alpha)^{i+1}}{\longrightarrow} e_{2} M\right)=\left\{\left(0,\left(0,0, \cdots, 0, b_{1}, \cdots, b_{j-(i+1)}\right)^{t}, 0\right) \mid b_{t} \in k, t=1, \cdots, j-(i+1)\right\} \text {. }
$$

We have $\operatorname{dim}_{k} \operatorname{Ext}_{\Lambda}^{1}\left(\left(S_{1}, \cdots, S_{1}\right)^{t}, M\right)=i+2-(j+i+1-n)=n+1-j$ for $j \leq n+1$.

Next, we compute the second Ext-group.

$$
\operatorname{Ext}_{\Lambda}^{2}\left(\left(S_{1}, \cdots, S_{1}\right)^{t}, M\right)=\operatorname{ker}\left(e_{3} M \stackrel{\gamma(\beta \alpha \gamma)^{i+1}}{\longrightarrow} e_{1} M\right) / \Im\left(e_{2} M \stackrel{\beta(\alpha \gamma \beta)^{n-i}}{\longrightarrow} e_{3} M\right) \text {. }
$$

We compute the kernel for

$$
d_{3}^{\times}:\left(e_{3} M \stackrel{\gamma(\beta \alpha \gamma)^{i+1}}{\longrightarrow} e_{1} M\right) .
$$

We have

$$
d_{3}^{\times}\left(0,0,\left(a_{1}, a_{2}, \cdots, a_{j}\right)^{t}\right)=\left(\left(0,0, \cdots, 0, a_{1}, \cdots, a_{j-(i+1)}\right)^{t}, 0,0\right),
$$

where $a_{1}$ is in the coordinate number $i+2$. This shows that

$$
\operatorname{ker}\left(e_{3} M \stackrel{\gamma(\beta \alpha \gamma)^{i+1}}{\longrightarrow} e_{1} M\right)=\left\{\left(0,0,\left(0,0, \cdots, 0, a_{1}, \cdots, a_{j}\right)^{t}\right) \mid a_{r} \in k, r=1, \cdots, j\right\},
$$

where $a_{1}$ is in the coordinate number $j-i$. We compute the image for

$$
d_{2}^{\times}:\left(e_{2} M \stackrel{\beta(\alpha \gamma \beta)^{n-i}}{\longrightarrow} e_{3} M\right) .
$$

We have

$$
d_{2}^{\times}\left(0,\left(b_{1}, b_{2}, \cdots, b_{s}, \cdots, b_{j}\right)^{t}, 0\right)=\left(0,0,\left(0,0, \cdots, 0, b_{1}, \cdots, b_{s}\right)^{t}\right),
$$

where $b_{1}$ is in the coordinate number $n-i+1$ and therefore $b_{s}$ is the coordinate number $n-i+s=j$ and $s=j+i-n$. This implies that

$$
\mathfrak{I}\left(e_{2} M \stackrel{\beta(\alpha \gamma \beta)^{n-i}}{\longrightarrow} e_{3} M\right)=\left\{\left(0,0,\left(0,0, \cdots, 0, b_{1}, \cdots, b_{j}\right)^{t}\right) \mid b_{t} \in k, t=1, \cdots, j\right\},
$$

where $b_{1}$ is in the coordinate number $n-i+1$. We have

$$
\operatorname{dim}_{k} \operatorname{Ext}_{\Lambda}^{2}\left(\left(S_{1}, \cdots, S_{1}\right)^{t}, M\right)=n-i+1-(j-i)=n+1-j
$$

for $j \leq n+1$. We compute the third Ext-group.

$$
\operatorname{Ext}_{\Lambda}^{3}\left(\left(S_{1}, \cdots, S_{1}\right)^{t}, M\right)=\operatorname{ker}\left(e_{1} M \stackrel{\alpha(\gamma \beta \alpha)^{n-i}}{\longrightarrow} e_{2} M\right) / \mathfrak{I}\left(e_{3} M \stackrel{\gamma(\beta \alpha \gamma)^{i+1}}{\longrightarrow} e_{1} M\right) \text {. }
$$


We compute the kernel for

$$
d_{4}^{\times}:\left(e_{1} M \stackrel{\alpha(\gamma \beta \alpha)^{n-i}}{\longrightarrow} e_{2} M\right) .
$$

We have

$$
d_{4}^{\times}\left(\left(a_{1}, a_{2}, \cdots, a_{s}, \cdots, a_{j+1}\right)^{t}, 0,0\right)=\left(0,\left(0,0, \cdots, 0, a_{1}, \cdots, a_{s}\right)^{t}, 0\right),
$$

where $a_{1}$ is in the coordinate number $n-i+1$ and therefore $a_{s}$ is in the coordinate number $n-i+s=j+1, s=j+i+1-n$ and consequently, $s+1=j+i+2-n$. Hence

$\operatorname{ker}\left(e_{1} M \stackrel{\alpha(\gamma \beta \alpha)^{n-i}}{\longrightarrow} e_{2} M\right)=\left\{\left(\left(0,0, \cdots, 0, a_{s+1}, \cdots, a_{j+1}\right)^{t}, 0,0\right) \mid a_{r} \in k, r=s+1, \cdots, j+1\right\}$.

We compute the image for

$$
d_{3}^{\times}:\left(e_{3} M \stackrel{\gamma(\beta \alpha \gamma)^{i+1}}{\longrightarrow} e_{1} M\right) .
$$

We have

$$
d_{3}^{\times}\left(0,0,\left(b_{1}, b_{2}, \cdots, b_{j}\right)^{t}\right)=\left(\left(0,0, \cdots, 0, b_{1}, \cdots, b_{j-i}\right)^{t}, 0,0\right),
$$

where $b_{1}$ is in the coordinate number $i+3$. This implies that

$$
\mathfrak{J}\left(e_{3} M \stackrel{\gamma(\beta \alpha \gamma)^{i+1}}{\longrightarrow} e_{1} M\right)=\left\{\left(\left(0,0, \cdots, 0, b_{1}, \cdots, b_{j-i}\right)^{t}, 0,0\right) \mid b_{t} \in k, t=1, \cdots, j-i\right\} .
$$

We therefore have $\operatorname{dim}_{k} \operatorname{Ext}_{\Lambda}^{3}\left(\left(S_{1}, \cdots, S_{1}\right)^{t}, M\right) i+3-(j+i+2-n)=n+1-j$ for $j \leq n+1$. We discuss the fourth Ext-group.

$$
\operatorname{Ext}_{\Lambda}^{4}\left(\left(S_{1}, \cdots, S_{1}\right)^{t}, M\right)=\operatorname{ker}\left(e_{2} M \stackrel{\beta(\alpha \gamma \beta)^{i+1}}{\longrightarrow} e_{3} M\right) / \Im\left(e_{1} M \stackrel{\alpha(\gamma \beta \alpha)^{n-i}}{\longrightarrow} e_{2} M\right) \text {. }
$$

We compute the kernel for

$$
d_{5}^{\times}:\left(e_{2} M \stackrel{\beta(\alpha \gamma \beta)^{i+1}}{\longrightarrow} e_{3} M\right) .
$$

We have

$$
d_{5}^{\times}\left(0,\left(a_{1}, a_{2}, \cdots, a_{j}\right)^{t}, 0\right)=\left(0,0,\left(0,0, \cdots, 0, a_{1}, \cdots, a_{j-i}\right)^{t}\right),
$$

where $a_{1}$ is in the coordinate number $i+2$. Hence

$$
\begin{aligned}
& \operatorname{ker}\left(e_{2} M \stackrel{\beta(\alpha \gamma \beta)^{i+1}}{\longrightarrow} e_{3} M\right) \\
& =\left\{\left(0,\left(0,0, \cdots, 0, a_{1}, \cdots, a_{j-(i+1)}\right)^{t}, 0\right) \mid a_{r} \in k, r=1, \cdots, j-(i+1)\right\},
\end{aligned}
$$

where $a_{1}$ is in the coordinate number $j-i$. We compute the image for

$$
d_{4}^{\times}:\left(e_{1} M \stackrel{\alpha(\gamma \beta \alpha)^{n-i}}{\longrightarrow} e_{2} M\right)
$$

to have

$$
d_{4}^{\times}\left(\left(b_{1}, b_{2}, \cdots, b_{s}, \cdots, b_{j}\right)^{t}, 0,0\right)=\left(0,\left(0,0, \cdots, 0, b_{1}, \cdots, b_{s}\right)^{t}, 0\right),
$$

where $b_{1}$ is in the coordinate number $n-i+1$ and therefore $b_{s}$ is in the coordinate number $n-i+s=j, s=j+i-n$. Consequently, $s+1=j+i+1-n$. 
This shows that

$$
\mathfrak{I}\left(e_{1} M \stackrel{\alpha(\gamma \beta \alpha)^{n-i}}{\longrightarrow} e_{2} M\right)=\left\{\left(0,\left(0,0, \cdots, 0, b_{1}, \cdots, b_{j-i}\right)^{t}, 0\right) \mid b_{t} \in k, t=1, \cdots, j\right\},
$$

where $b_{1}$ is in the coordinate number $n-i+1$. We have

$$
\operatorname{dim}_{k} \operatorname{Ext}_{\Lambda}^{4}\left(\left(S_{1}, \cdots, S_{1}\right)^{t}, M\right)=n-i+1-(j-i)=n+1-j
$$

for $j \leq n+1$. We discuss the fifth Ext-group.

$$
\operatorname{Ext}_{\Lambda}^{5}\left(\left(S_{1}, \cdots, S_{1}\right)^{t}, M\right)=\operatorname{ker}\left(e_{3} M \stackrel{\gamma(\beta \alpha \gamma)^{n-i}}{\longrightarrow} e_{1} M\right) / \mathfrak{T}\left(e_{2} M \stackrel{\beta(\alpha \gamma \beta)^{i+1}}{\longrightarrow} e_{3} M\right) \text {. }
$$

We compute the kernel for

$$
d_{6}^{\times}:\left(e_{3} M \stackrel{\gamma(\beta \alpha \gamma)^{n-i}}{\longrightarrow} e_{1} M\right) .
$$

We have

$$
d_{6}^{\times}\left(0,0,\left(a_{1}, a_{2}, \cdots, a_{s}, \cdots, a_{j}\right)^{t}\right)=\left(\left(0,0, \cdots, 0, a_{1}, \cdots, a_{s}\right)^{t}, 0,0\right),
$$

where $a_{1}$ is in the coordinate number $n-i+2$ and therefore $a_{s}$ is in the coordinate number $n-i+1+s=j, s=j+i-1-n$ and consequently, $s+1=j+i-n$. This shows that

$\operatorname{ker}\left(e_{3} M \stackrel{\gamma(\beta \alpha \gamma)^{n-i}}{\longrightarrow} e_{1} M\right)=\left\{\left(0,0,\left(0,0, \cdots, 0, a_{s+1}, \cdots, a_{j}\right)^{t}\right) \mid a_{r} \in(k, r=s+1, \cdots, j)\right\}$.

We compute the image for

$$
d_{5}^{\times}:\left(e_{2} M \stackrel{\beta(\alpha \gamma \beta)^{i+1}}{\longrightarrow} e_{3} M\right) .
$$

We have

$$
d_{5}^{\times}\left(0,\left(b_{1}, b_{2}, \cdots, b_{j}\right)^{t}, 0\right)=\left(0,0,\left(0,0, \cdots, 0, b_{1}, \cdots, b_{j-i}\right)^{t}, 0,0\right),
$$

where $b_{1}$ is in the coordinate number $i+2$. Hence

$$
\mathfrak{J}\left(e_{2} M \stackrel{\beta(\alpha \gamma \beta)^{i+1}}{\longrightarrow} e_{3} M\right)=\left\{\left(0,0,\left(0,0, \cdots, 0, b_{1}, \cdots, b_{j-i}\right)^{t}\right) \mid b_{t} \in k, t=1, \cdots, j\right\} .
$$

We therefore have

$$
\operatorname{dim}_{k} \operatorname{Ext}_{\Lambda}^{5}\left(\left(S_{1}, \cdots, S_{1}\right)^{t}, M\right)=i+2-(j+i-n)=n+2-j \text { for } j \leq n+2 .
$$

$\operatorname{dim}_{k} \operatorname{Ext}_{\Lambda}^{t}(3 i+1,3 j+1)=n+1-j$ for $t=1,2,3$ and 4 , and $n+2-j$ for $t=5$. We therefore have $n+1=j$ and $n+2=j$.

It follows from the fifth Ext-group that the condition $\operatorname{Ext}_{\Lambda}^{n}(M, N)=0$ for $n \gg 0 \Leftrightarrow \operatorname{Ext}_{\Lambda}^{n}(N, M)=0$ for $n \gg 0$ holds.

\section{Conclusion}

The study found that the Ext-groups of pairs $(M, N)$ of modules over Nakayama algebras of type $(n, n, n)$ satisfy the condition $\operatorname{Ext}_{\Lambda}^{n}(M, N)=0$ for $n \gg 0 \Leftrightarrow \operatorname{Ext}_{\Lambda}^{n}(N, M)=0$ for $n \gg 0$ and justified the claim with projectives of lengths $3 n+1$ and $3 n+2$ by using combinations of modules of different lengths. 


\section{Acknowledgements}

We are grateful to the referee for many useful suggestions.

\section{References}

[1] Schröer, J. (2016) Nakayama Algebras, 2016. www.math.un-bonn.de/people/Schroer/fd-atlas/FD-NakayamaAlgebras.pdf

[2] Rotman, J.J. (2009) An Introduction to Homological Algebra. 2nd Edition, Universitex, Springer.

[3] Auslander, M., Reiten, I. and Smalø, S. (1995) Representation Theory of Artin Algebras. Series: Cambridge Studies in Advanced Mathematics (No. 36). Cambridge University Press, Cambridge.

\section{Scientific Research Publishing}

Submit or recommend next manuscript to SCIRP and we will provide best service for you:

Accepting pre-submission inquiries through Email, Facebook, LinkedIn, Twitter, etc. A wide selection of journals (inclusive of 9 subjects, more than 200 journals)

Providing 24-hour high-quality service

User-friendly online submission system

Fair and swift peer-review system

Efficient typesetting and proofreading procedure

Display of the result of downloads and visits, as well as the number of cited articles

Maximum dissemination of your research work

Submit your manuscript at: http://papersubmission.scirp.org/

Or contact jamp@scirp.org 